\title{
Negative ion behavior in single- and dual-frequency plasma etching reactors: Particle-in-cell/Monte Carlo collision study
}

\author{
V. Georgieva* and A. Bogaerts \\ PLASMANT Research Group, Department of Chemistry, University of Antwerp, Universiteitsplein 1, 2610 Wilrijk-Antwerp, Belgium
}

(Received 18 November 2005; revised manuscript received 4 January 2006; published 6 March 2006)

\begin{abstract}
Particle-in-cell/Monte Carlo simulations are used to simulate the trajectories and energies of randomly sampled $\mathrm{F}^{-}$and $\mathrm{CF}_{3}^{-}$ions in capacitively coupled radio-frequency discharges, in order to clarify the movement of the negative ions in the sheaths and plasma in both single- and dual-frequency regimes, as well as in symmetric and asymmetric discharges. In the single-frequency reactor both types of negative ions are confined in the plasma and the main loss mechanism is recombination with positive ions. In the dual-frequency reactor under certain operating conditions when the sheaths are wide and the bulk plasma is narrow the light $\mathrm{F}^{-}$ions move across from one sheath to the other, and they can even be lost at the electrodes. The main loss mechanisms are then electron detachment and absorption at the electrodes. The much heavier $\mathrm{CF}_{3}^{-}$ions are still confined in the bulk and represent the major negative charge. In an asymmetric discharge the electric field in the sheath to the smaller (powered) electrode is much stronger than that in the sheath to the grounded electrode. Consequently, the $\mathrm{F}^{-}$ions reach mainly the grounded electrode.
\end{abstract}

DOI: 10.1103/PhysRevE.73.036402

PACS number(s): 52.27.Cm, 52.65.Rr, 52.65.Pp

\section{INTRODUCTION}

In single-frequency capacitively coupled radio-frequency (rf) discharges the ion density profile is typically constant throughout the rf cycle since the ions cannot respond to the rapidly oscillating electric field. For example, if the applied rf is $13.56 \mathrm{MHz}$, i.e., in the order of $10^{7} \mathrm{~Hz}$, the calculated electron plasma frequency $f_{p e}=\omega_{p e} / 2 \pi$, is in the order of $10^{8} \mathrm{~Hz}$, while the ion plasma frequency $f_{p i}=\omega_{p i} / 2 \pi$ is only in the order of $10^{6} \mathrm{~Hz}$. The electron plasma frequency $\omega_{p e}$ and ion plasma frequency $\omega_{p i}$ are defined by [1]

$$
\begin{aligned}
& \omega_{p e}=\left(\frac{e^{2} n_{e}}{\varepsilon_{0} m}\right)^{1 / 2}, \\
& \omega_{p i}=\left(\frac{e^{2} n_{i}}{\epsilon_{0} M}\right)^{1 / 2} \quad \text { (for a singly charged ion) }
\end{aligned}
$$

where $n_{e}$ and $n_{i}$ are the electron and ion number density in the bulk plasma, $m$ and $M$ are the electron and ion mass, respectively, $e$ is the elementary charge, and $\varepsilon_{0}$ is the vacuum permittivity. The electric fields within the sheaths established at steady state point from the plasma to the electrodes. Consequently, the electrostatic force acting on the negative ions is directed into the plasma and it confines them there, while the positive ions enter the sheaths and are accelerated to the electrodes. This is confirmed by numerical results of the charged particle density distributions in fluorocarbon-based discharges sustained in conventional single-frequency (13.56 MHz) reactors [1-6].

In dual-frequency reactors, however, under certain operating conditions the ions can respond to the electric field because the applied second, low-frequency (LF), for example

*Electronic address: violeta.georgieva@ua.ac.be
$2 \mathrm{MHz}$, i.e., $2 \times 10^{6} \mathrm{~Hz}$, is close to the ion plasma frequency. As is observed in the simulation results presented in Ref. [2] for an $\mathrm{Ar} / \mathrm{CF}_{4} / \mathrm{N}_{2}$ discharge, the positive ion density profile depends slightly on time in the LF cycle. The $\mathrm{F}^{-}$ion density distribution, on the other hand, was found to be considerably modulated throughout the LF cycle. The light $\mathrm{F}^{-}$ions respond more or less to the applied electric field and move across from one sheath to the other, and they can even be lost at the electrodes, which is an unexpected phenomenon. For example, a negative ion flux at the electrodes is numerically predicted [7] and experimentally observed [8] in a pulsed dual-frequency capacitively coupled discharge during the off phase, which could be logically anticipated. However, in the present dual-frequency reactor the negative ions are detected at the electrodes when the power source is active. In this case the much heavier $\mathrm{CF}_{3}^{-}$ions are still confined in the bulk and represent the major negative charge. The $\mathrm{F}^{-}$flux at the electrodes can vary significantly depending on the reactor parameters, i.e., applied frequencies and voltage amplitudes [9].

The negative charge injection can reduce the local charging at the electrodes, which is essential for establishing charge-free plasma processes for etching. Consequently, a systematic study of the negative ion behavior in electronegative discharges, such as in $\mathrm{CF}_{4}$ mixtures, is important for further improvement of the etching process. The purpose of the present paper is to clarify the movement of the negative ions in the sheaths and plasma and the energy they gain or lose in these regions. The trajectories and energies of randomly sampled $\mathrm{F}^{-}$and $\mathrm{CF}_{3}^{-}$ions are presented in both singleand dual-frequency regimes. The influence of operating conditions on the negative ion flux is also presented. The computation is based on a one-dimensional coordinate space and three-dimensional velocity space particle-in-cell/Monte Carlo collision (PIC/MCC) algorithm [2,6]. The simulations have been carried out for both symmetric and asymmetric discharges. 

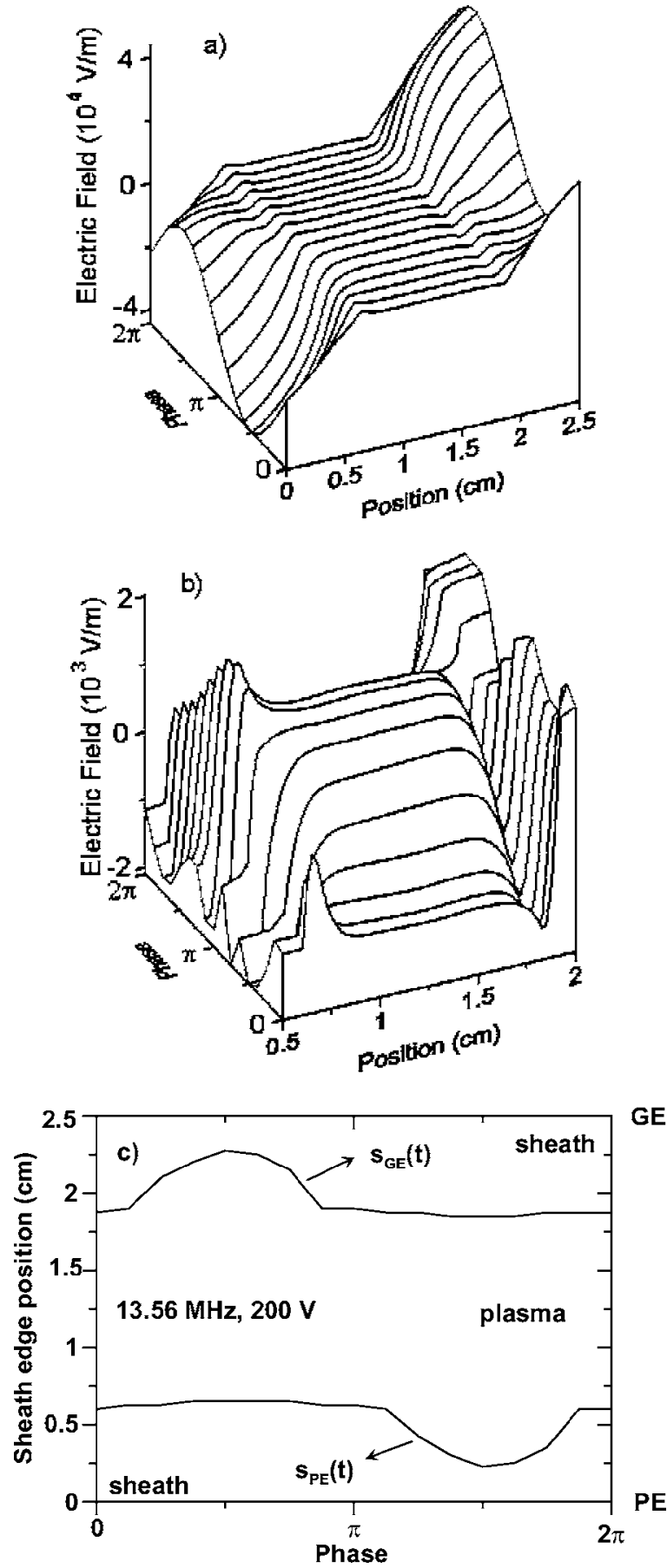

FIG. 1. Calculated electric field distribution in the entire discharge (a) and in the plasma region (b) as well as the calculated sheath edge position in front of the PE and GE (c), as a function of time in one rf cycle. A pure $\mathrm{CF}_{4}$ discharge is sustained in a 13.56 MHz reactor, at an applied voltage of $200 \mathrm{~V}$, a pressure of $200 \mathrm{mTorr}$, and an electrode distance of $2.5 \mathrm{~cm}$.

In Sec. II the outlines of the model and the input parameters are presented. In Sec. III the results of the simulations, i.e., the electric field distribution as a function of time in the LF or rf cycle, instantaneous sheath thickness, and trajectories and energies of the negative ions are shown and discussed. Finally, a summary is given in Sec. IV.

\section{DESCRIPTION OF THE MODEL}

The plasma is sustained between two parallel plates, each $20 \mathrm{~cm}$ in diameter and separated from each other by 2 or $2.5 \mathrm{~cm}$. One of the electrodes is driven by a single- or dual-frequency voltage source, i.e., in the latter case the applied voltage is the sum of the high-frequency (HF) and LF voltages

$$
V=V_{H F} \sin \left(\omega_{H F} t\right)+V_{L F} \sin \left(\omega_{L F}, t\right),
$$

where $V_{H F}$ and $V_{L F}$ are the HF and LF voltage amplitudes, and $\omega_{H F}$ and $\omega_{L F}$ are the applied HF and LF. The other electrode is grounded. The computation is based on a onedimensional coordinate space and three-dimensional velocity space PIC/MCC algorithm [10,11].

The PIC method deals with charged particles moving around due to the forces they generate and the applied fields $[10,11]$. The particle simulation can be done with a small number of particles, which is many orders of magnitude lower than in a real plasma. Each computer particle or socalled superparticle represents for example $10^{6}-10^{9}$ real particles. The method uses classical mechanics for the equations of motion, i.e.,

$$
\begin{gathered}
m \frac{d \mathbf{v}}{d t}=\mathbf{F}, \\
\frac{d \mathbf{x}}{d t}=\mathbf{v},
\end{gathered}
$$

to advance the particles to new positions and velocities. In the electrostatic case Poisson's equation is solved based on the charged particle densities and then the electric field is calculated. The charged species that are followed in the present model for an $\mathrm{Ar} / \mathrm{CF}_{4} / \mathrm{N}_{2}$ discharge are electrons, $\mathrm{Ar}^{+}, \mathrm{CF}_{3}^{+}, \mathrm{N}_{2}^{+}, \mathrm{F}^{-}$, and $\mathrm{CF}_{3}^{-}$ions. The neutral gas is assumed to be uniformly distributed. The collisions between the particles are treated by a MCC procedure, an ion-molecule collision model for endothermic reactions, and a $\mathrm{MC}$ simulation of positive-negative ion and electron-positive ion recombination collisions for a given rate constant. The choice of the charged particles followed in the model, the collisions between them and with neutral species, and all data and techniques used to calculate the collision probabilities are presented and discussed in detail elsewhere (see Refs. [2,6,12]). The model is developed in Cartesian coordinates in order to simulate symmetric discharges $[2,6,12]$ and in spherical coordinates in order to simulate asymmetric discharges $[13,14]$.

Based on Eqs. (4) and (5) the new velocity, consequently energy, and position of randomly sampled $\mathrm{F}^{-}$and $\mathrm{CF}_{3}^{-}$ions are saved in every ion time step. Since the much heavier ions hardly move at one electron time step, different electron and ion time steps are applied in the model in order to speed up the calculations [15]. The ion time step is set up to 50 times higher than the electron time step in the present model. If the sampled ion participates in a collision the postcollision velocity is calculated. The ion is followed until it is lost. As was found in previous simulations in single-frequency reactors, the main loss mechanism is recombination with positive ions in the plasma $[5,6]$. Electron detachment, which mainly 

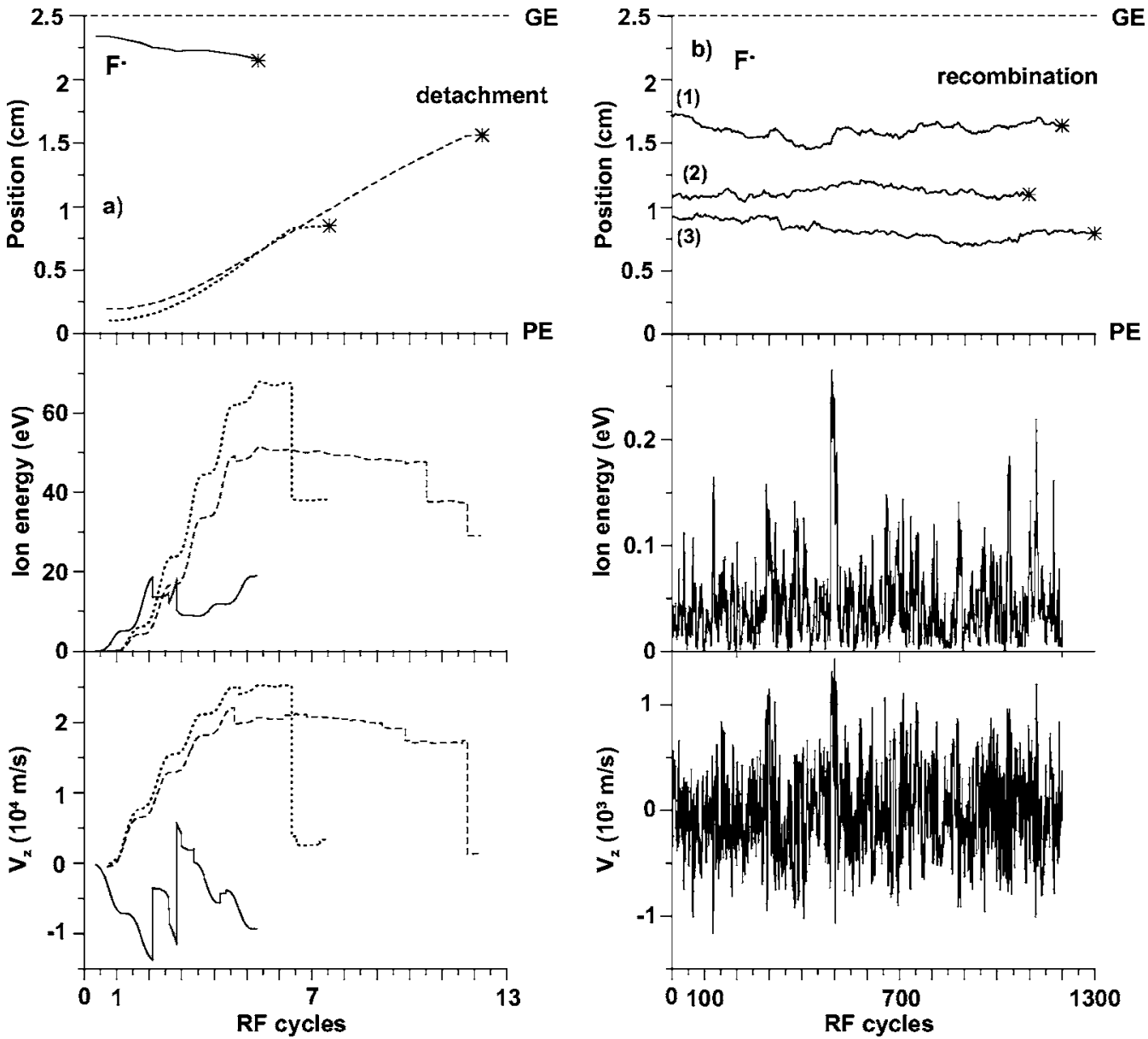

FIG. 2. Calculated position, energy, and $z$ velocity of randomly sampled $\mathrm{F}^{-}$ions, created in electron attachment processes, as a function of time represented by rf cycles $\left(\tau_{r f}=7.4 \times 10^{-8} \mathrm{~s}\right)$. The ions are lost in electron detachment (a) or in recombination (b) collisions (asterisk). The positions of the PE and GE are also shown. The operating conditions are the same as in Fig. 1.

takes place in the sheaths, has significantly lower reaction rates in comparison with recombination $[5,6]$. All reaction processes of $\mathrm{F}^{-}$and $\mathrm{CF}_{3}^{-}$ions with $\mathrm{CF}_{4}$ including electron detachment, which are considered in the model, are presented in Ref. [6] along with their threshold energies. The corresponding analytical cross sections calculated from the ion-molecule collision model for endothermic reactions are presented in Ref. [12]. It is helpful to mention here that the lowest threshold energy for an electron detachment process of $\mathrm{F}^{-}$is $3.521 \mathrm{eV}$, and of $\mathrm{CF}_{3}^{-}$is $1.871 \mathrm{eV}$. In the dualfrequency regime a third loss mechanism appears to be possible, which is absorption of the negative ions at the electrodes. A sufficient number of ions have been traced at different simulation times so that the trajectories and energies of the negative ions presented below can be considered representative.

\section{RESULTS AND DISCUSSION}

The operating conditions in the single-frequency 13.56 MHz regime are the same as in Ref. [6] for a pure $\mathrm{CF}_{4}$ discharge: gas pressure of $200 \mathrm{mTorr}$, electrode distance of $2.5 \mathrm{~cm}$, and applied voltage amplitude of $200 \mathrm{~V}$. The operating conditions for the symmetric and asymmetric
$27+2 \mathrm{MHz}$ regime are the same as in Refs. [9] and [14] , that is, the applied HF and LF voltages are 200 and $400 \mathrm{~V}$, respectively, for a gas mixture $\mathrm{Ar} / \mathrm{CF}_{4} / \mathrm{N}_{2}$ at a ratio of 0.8 / $0.1 / 0.1$, a gas pressure of $30 \mathrm{mTorr}$, and an electrode distance of $2 \mathrm{~cm}$. In order to show the dependence of the flux on the reactor parameters, negative ions have been traced also in $40+2,60+2$, and $100+2 \mathrm{MHz}$ regimes at $\mathrm{HF}$ and $\mathrm{LF}$ voltage amplitudes of 200 and $400 \mathrm{~V}$, respectively. The neutral gas temperature is set to $300 \mathrm{~K}(0.026 \mathrm{eV})$.

The figures presented below show the calculated position, particle energy, and velocity component in the $z$ or $r$ direction, depending on the coordinate system, which is considered, i.e., Cartesian for the symmetric reactor and spherical for the asymmetric reactor, at every ion time step as a function of time represented by a LF or rf cycle.

\section{A. Single-frequency reactors}

A pure $\mathrm{CF}_{4}$ discharge sustained in a single-frequency reactor is considered at the operating conditions described above. The calculated electric field distribution in the entire discharge is presented in Fig. 1(a). The powered and grounded electrodes are positioned at 0 and $2.5 \mathrm{~cm}$, respectively. The electric field in the sheaths has maximum value in 


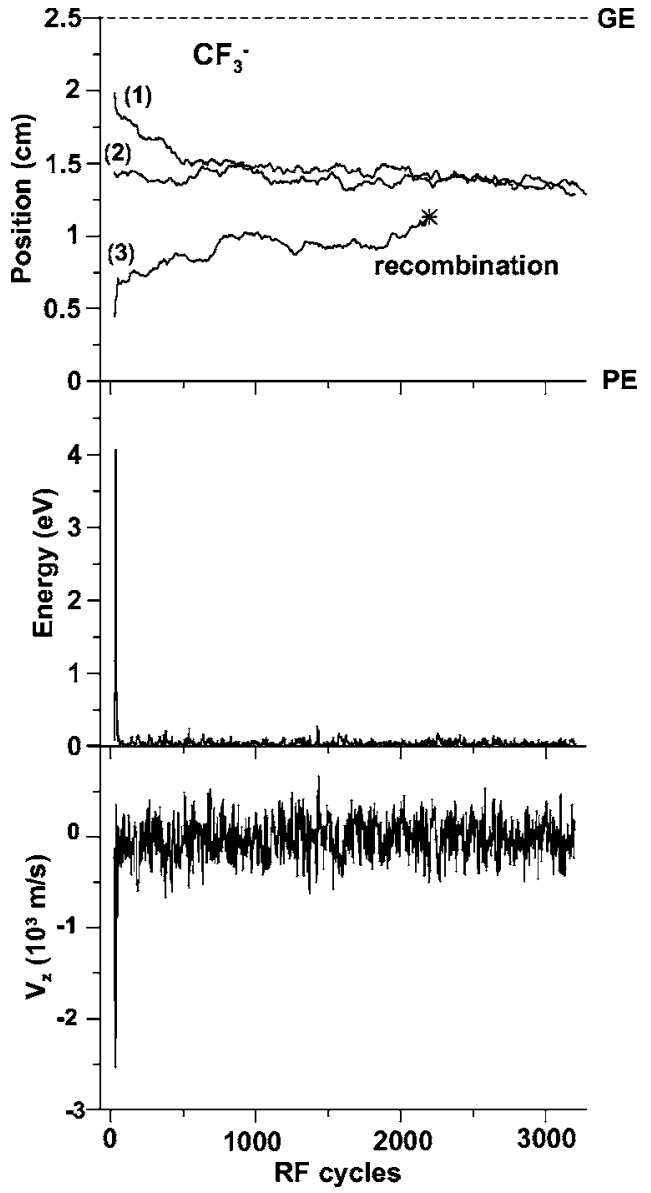

FIG. 3. Calculated position, energy, and $z$ velocity of randomly sampled $\mathrm{CF}_{3}^{-}$ions, created in electron attachment collisions, as a function of time represented by rf cycles $\left(\tau_{r f}=7.4 \times 10^{-8} \mathrm{~s}\right)$. The $\mathrm{CF}_{3}^{-}$ions 1 and 2 have not been lost during the time of observation; the ion 3 is lost in recombination. The operating conditions are the same as in Fig. 1.

the order of $4.5 \times 10^{4} \mathrm{~V} / \mathrm{m}$. Since the profile in the bulk plasma cannot be seen clearly in Fig. 1(a), a close look at the electric field in the bulk plasma is presented in Fig. 1(b), at positions between 0.5 and $2 \mathrm{~cm}$, i.e., the sheaths are excluded [compare with Fig. 1(c)]. It shows that the electric field reaches values up to $2 \times 10^{3} \mathrm{~V} / \mathrm{m}$. The comparatively strong electric field in the bulk plasma and the observed double-layer structure and field reversal at the bulk-sheath interface are typical for electronegative discharges [Fig. 1(b)]. The electric field in the sheaths is directed from the plasma to the electrodes. Consequently, if a negative ion is created in the sheath, for example, after an electron attachment collision, it will be accelerated toward the plasma and gain energy from the electric field [see Fig. 2(a)]. This explains the comparatively high averaged $\mathrm{F}^{-}$and $\mathrm{CF}_{3}^{-}$ion energies up to $8 \mathrm{eV}$, calculated in the sheaths [6]. Figure 1(c) presents the calculated sheath edge position in front of the powered (PE) and grounded (GE) electrodes, $s_{P E}(t)$ and $s_{G E}(t)$, respectively, as a function of the phase in the rf cycle. This figure is included in order to give an idea about the plasma and sheath regions for the considered operating conditions. The sheath edge position is defined at the grid point where the quasineutrality is broken.
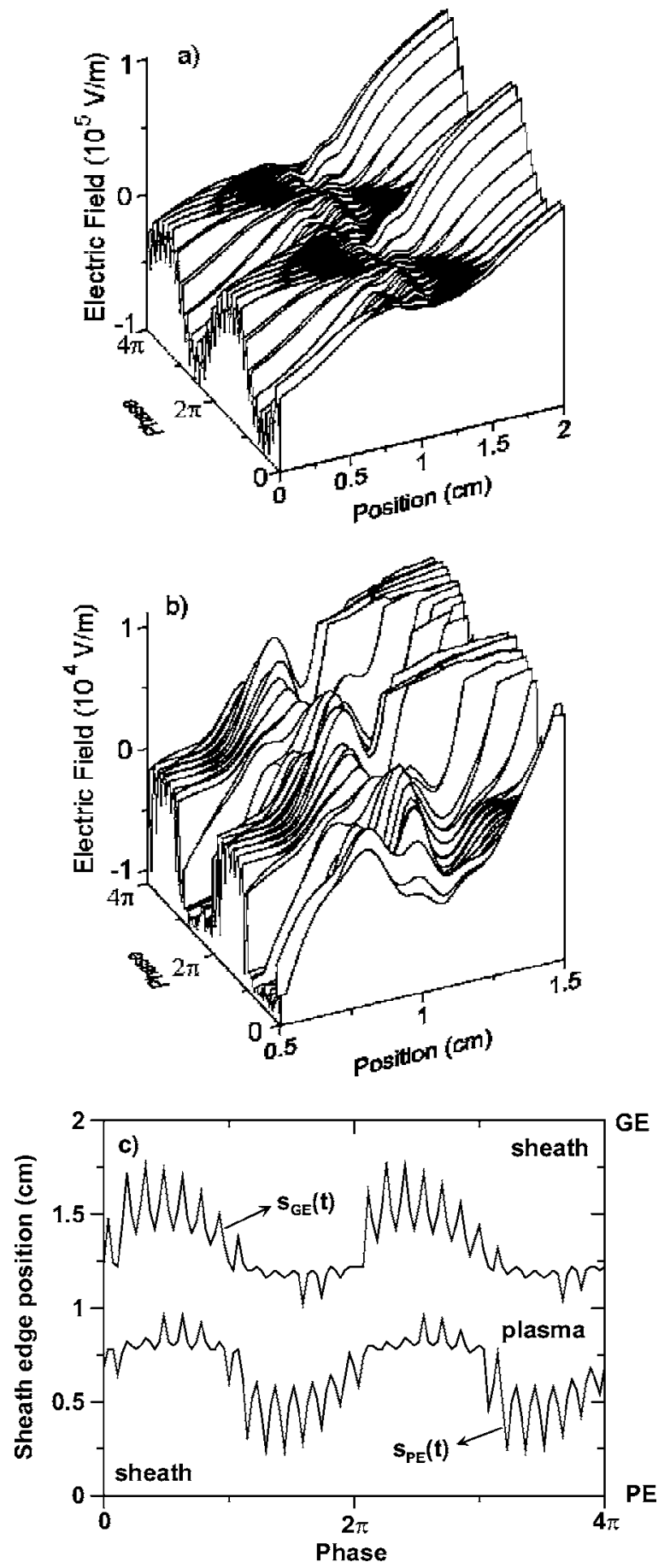

FIG. 4. Calculated electric field distribution in the entire discharge (a) and in the plasma region (b), as well as the calculated sheath edge position in front of the PE and GE (c), as a function of time in two LF cycles. A symmetric $\mathrm{Ar} / \mathrm{CF}_{4} / \mathrm{N}_{2}$ discharge is sustained in a dual-frequency $27+2 \mathrm{MHz}$ reactor, at applied $\mathrm{HF}$ and LF voltages of 200 and $400 \mathrm{~V}$, respectively, a pressure of $30 \mathrm{mTorr}$, and an electrode distance of $2 \mathrm{~cm}$.

Figure 2(a) presents the calculated position, energy, and velocity in the $z$ direction of randomly sampled $\mathrm{F}^{-}$ions created by electron attachment collisions in the sheaths and lost in electron detachment collisions, as a function of time, represented by rf cycles. The positions of the PE and GE are 

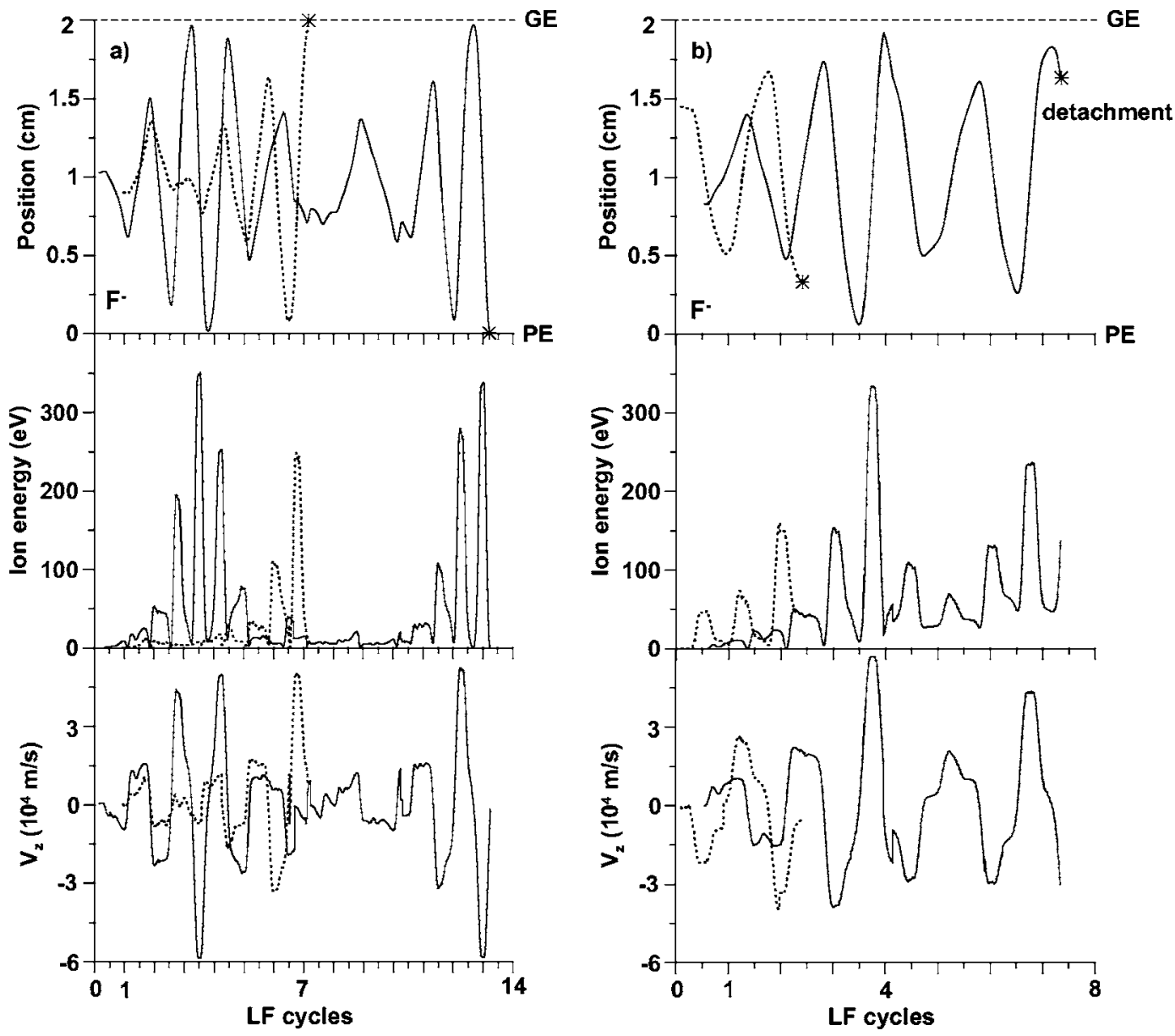

FIG. 5. Calculated position, energy, and $z$ velocity of randomly sampled $\mathrm{F}^{-}$ions, created in electron attachment processes, as a function of time represented by LF cycles $\left(\tau_{L F}=5 \times 10^{-7} \mathrm{~s}\right)$. The ions are lost at the electrodes (a) or in electron detachment collisions (b). The operating conditions are the same as in Fig. 4.

denoted at 0 and $2.5 \mathrm{~cm}$, respectively. The ions are sampled at different times during the simulation. While traveling from the sheath to the plasma the ions gain sufficiently high energy and they can participate in reactive collisions with corresponding threshold energies or in elastic collisions [see the abrupt changes in the energy and velocity of the ions presented in Fig. 2(a)]. If the reactive collision is an electron detachment, the ion disappears (denoted with an asterisk). The lifetime of $\mathrm{F}^{-}$ions created in the sheaths is typically less than $10 \mathrm{rf}$ cycles, i.e., $7 \times 10^{-7} \mathrm{~s}$ for a rf of $13.56 \mathrm{MHz}$.

Figure 2(b) shows the trajectory, energy, and velocity in the $z$ direction of randomly sampled $\mathrm{F}^{-}$ions created by electron attachment collisions in the bulk plasma and lost by recombination. The energy and $z$ velocity are only presented for one of the ions, denoted (1), since the corresponding values of the other two ions are in the same range. The electric field in the bulk plasma is weak and the ions move slowly, participating only in elastic collisions, with neutrals. Consequently, their energy does not change considerably. The calculated averaged $\mathrm{F}^{-}$ion energy in the bulk plasma is around $0.04 \mathrm{eV}$, i.e., close to the neutral gas temperature [6]. It can be seen that the lifetime of the $\mathrm{F}^{-}$ions is now more than $1000 \mathrm{rf}$ cycles, i.e., above $7 \times 10^{-5} \mathrm{~s}$ for a rf of 13.56 MHz, which explains the long computational time needed to reach convergence in PIC/MCC simulations of electronegative discharges. Although the recombination frequency is low, this charge neutralization process is the main loss mechanism of the negative ions. Only around $1.5 \%$ of all $\mathrm{F}^{-}$ions, lost during the time of simulation, disappeared in electron detachment collisions. Indeed, negative ions are almost absent in the sheaths. The few ions presented there are created by electron attachment collisions and they gain sufficient energy while moving to the bulk plasma, therefore, they participate in reactive collisions. Often these ions are lost in electron detachment collisions.

The same conclusions are reached for the $\mathrm{CF}_{3}^{-}$behavior, only its lifetime is longer. Figure 3 presents the position, energy, and $z$-velocity component of randomly sampled $\mathrm{CF}_{3}^{-}$ ions, as a function of time, represented by rf cycles. The energy and $z$ velocity of only one of the ions, denoted with (1), are shown since the corresponding values of the other two ions are in the same range. The ions are created by electron attachment collisions and at the beginning their energy is close to the neutral gas energy. If an ion is created in the sheath or at the sheath-plasma interface, like ions 1 and 3 , it moves slowly toward the center. Such an ion initially gains some energy from the electric field from a few $\mathrm{eV}$ to a few tens of eV, depending on the position in the sheath where 


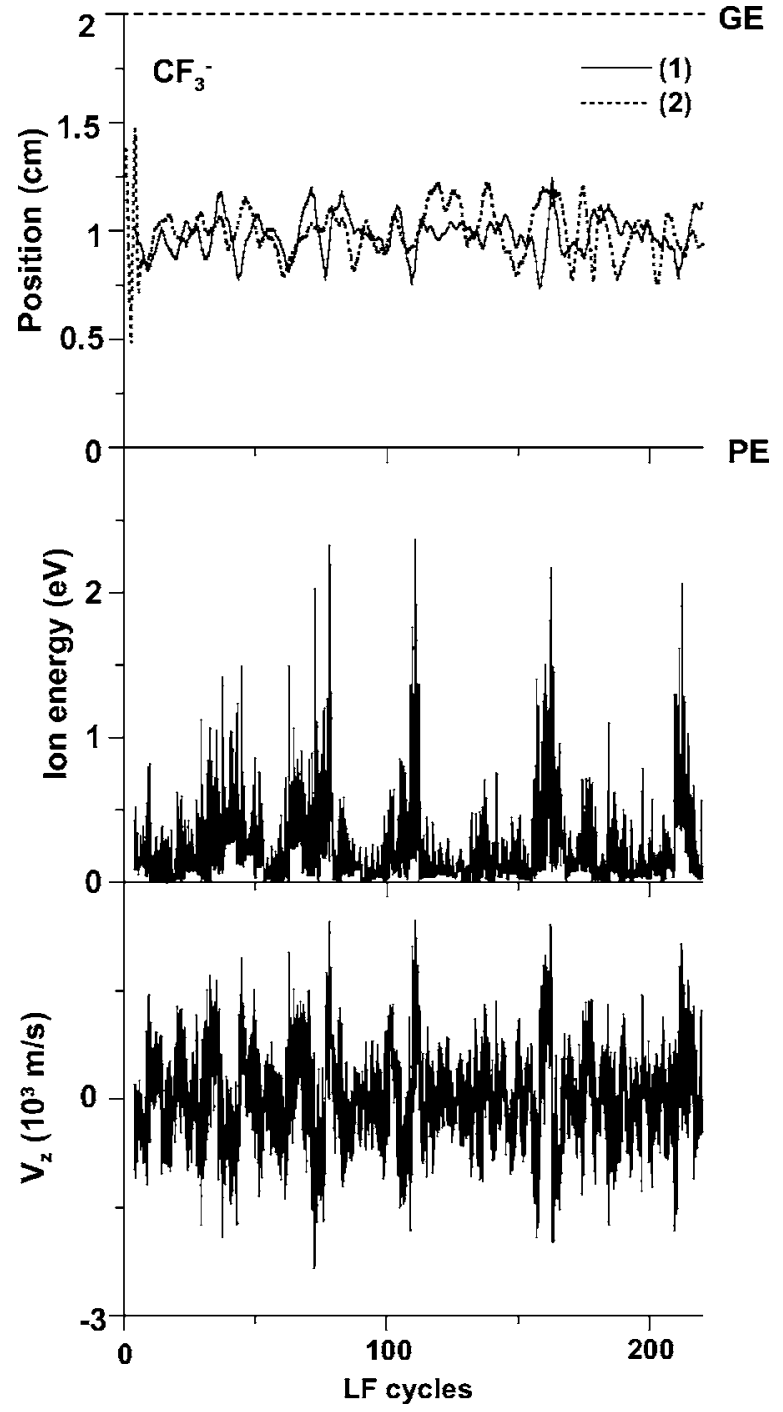

FIG. 6. Calculated position, energy, and $z$ velocity of randomly sampled $\mathrm{CF}_{3}^{-}$ions, created in electron attachment collisions, as a function of time represented by LF cycles $\left(\tau_{L F}=5 \times 10^{-7} \mathrm{~s}\right)$. The two ions have not been lost during the time of observation. The operating conditions are the same as in Fig. 4.

it is created. This energy is dissipated in a short time by the collisions with neutrals. If an ion is created in the plasma center, like the ion 2 , its maximum energy does not exceed a few times $0.1 \mathrm{eV}$. The calculated averaged $\mathrm{CF}_{3}^{-}$ion energy in the bulk plasma is around $0.04 \mathrm{eV}$ [6]. Again, like for $\mathrm{F}^{-}$ ions, recombination with positive ions in the plasma is the main loss mechanism of $\mathrm{CF}_{3}^{-}$ions, and its rate is low. As can be seen the ions 1 and 2 stay in the plasma for more than $3000 \mathrm{rf}$ cycles and recombination has not taken place yet. Ion 3 gets lost by recombination after 2200 rf cycles. Electron detachment of $\mathrm{CF}_{3}^{-}$ions takes place mainly in the sheaths, where the $\mathrm{CF}_{3}^{-}$ions gain sufficiently high energy to participate in reactive collisions, but the probability is low. Less than $1 \%$ of the $\mathrm{CF}_{3}^{-}$ions are lost in electron detachment collisions.

The $\mathrm{F}^{-}$and $\mathrm{CF}_{3}^{-}$ions have the same behavior in the asymmetric discharge sustained in a single-frequency reactor, i.e., they are confined in the plasma.

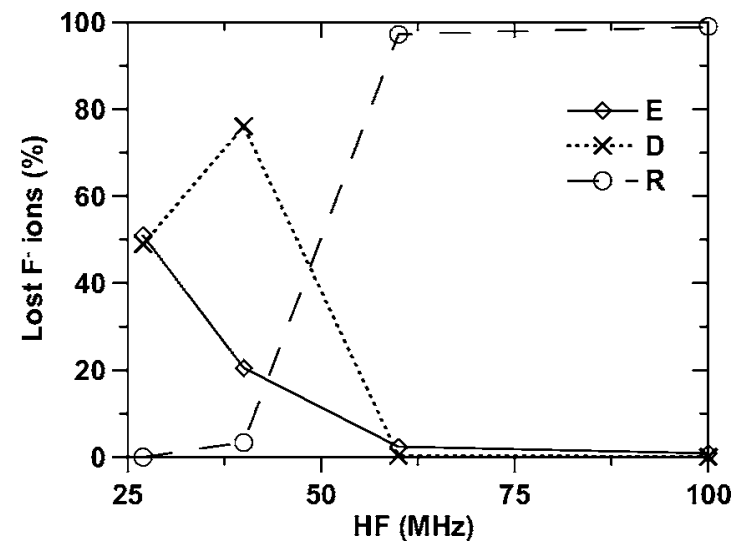

FIG. 7. Calculated percentage of the $\mathrm{F}^{-}$ions lost at the electrodes (solid line, E), or by electron detachment (dotted line, D), or recombination (dashed line, $\mathrm{R}$ ) in symmetric $\mathrm{Ar} / \mathrm{CF}_{4} / \mathrm{N}_{2}$ discharges sustained in the $27+2,40+2,60+2$, and $100+2 \mathrm{MHz}$ regimes, at applied HF and LF voltage amplitudes of 200 and $400 \mathrm{~V}$, respectively, a pressure of $30 \mathrm{mTorr}$, and an electrode distance of $2 \mathrm{~cm}$.

\section{B. Dual-frequency reactors}

As observed in Refs. [2] and [9], in the dual-frequency regime the $\mathrm{F}^{-}$ions can reach the electrodes and consequently, reduce the local charging. Figure 4(a) presents the calculated electric field distribution in a symmetric $\mathrm{Ar} / \mathrm{CF}_{4} / \mathrm{N}_{2}$ discharge sustained in a dual-frequency 27 $+2 \mathrm{MHz}$ reactor. The applied HF and LF voltages are 200 and $400 \mathrm{~V}$, respectively, and the gas pressure is $30 \mathrm{mTorr}$. The powered and grounded electrodes are positioned at 0 and $2 \mathrm{~cm}$, respectively. Similar to the single-frequency case, presented above, the electric field in the bulk plasma is shown separately in Fig. 4(b) because its profile and values cannot be seen clearly in Fig. 4(a). The maximum electric field in the sheaths is now in the order of $10^{5} \mathrm{~V} / \mathrm{m}$ [Fig. 4(a)] and in the bulk plasma it is in the order of $10^{4} \mathrm{~V} / \mathrm{m}$ [Fig. 4(b)]. Indeed, at a HF of $27 \mathrm{MHz}$ and when the applied LF voltage amplitude is comparable to or higher than the $\mathrm{HF}$ voltage amplitude, the behavior of the $\mathrm{Ar} / \mathrm{CF}_{4} / \mathrm{N}_{2}$ discharge is strongly electronegative (see Ref. [9] for details). The electric field in the plasma is then strong and double layers appear at the bulk-sheath interfaces. In the presence of the LF

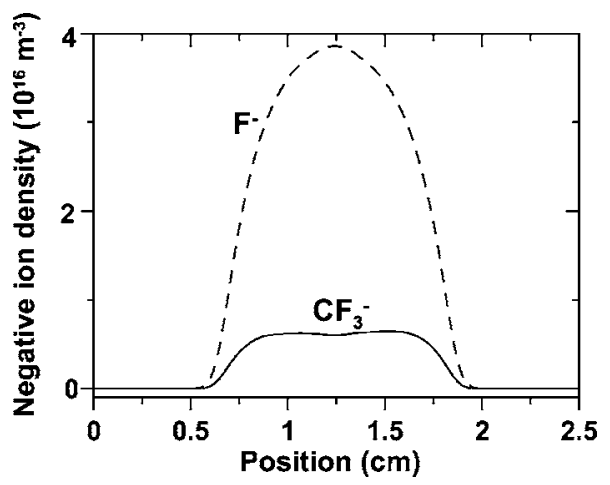

FIG. 8. Calculated $\mathrm{F}^{-}$and $\mathrm{CF}_{3}^{-}$density distributions in a singlefrequency reactor. The profiles are constant throughout the rf cycle. The operating conditions are the same as in Fig. 1. 

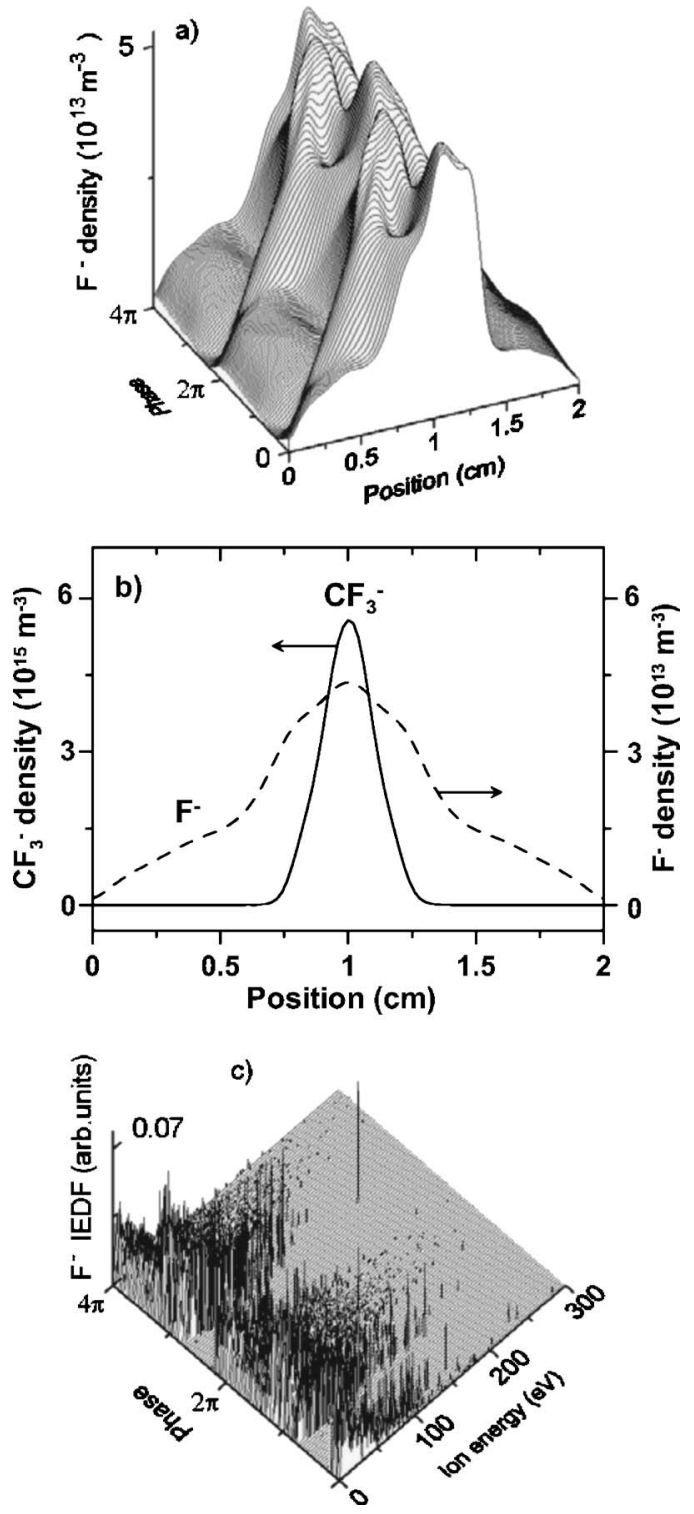

FIG. 9. Calculated $\mathrm{F}^{-}$density distribution as a function of time in two LF cycles (a) and averaged over two LF cycles (b, dashed line), $\mathrm{CF}_{3}^{-}$density distribution, the profile of which is constant throughout the LF cycle (b, solid line), and the energy distribution of the $\mathrm{F}^{-}$ions lost at the electrodes (c). The operating conditions are the same as in Fig. 4.

the $\mathrm{F}^{-}$ions respond more or less to the time variation of the electric field and are not confined in the bulk plasma. The sheaths oscillate with the applied frequencies [see Fig. 4(c)] and the sheath width is large enough; therefore $\mathrm{F}^{-}$ions gain considerable energy when they move toward the plasma for some time in the LF cycle so that they can traverse the narrow bulk plasma and continue to the other sheath. The electric field there retards the ions until they change their direction again to the center. As a result, the $\mathrm{F}^{-}$ions can oscillate from one sheath to the other and after several LF cycles some of them can reach the electrodes.

Figure 5(a) presents the position, energy, and z-velocity component of randomly sampled $\mathrm{F}^{-}$ions, which are lost at the electrodes, as a function of time, represented by LF

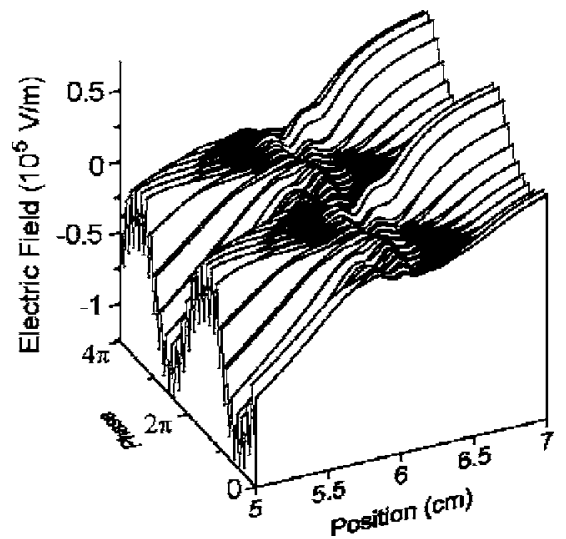

FIG. 10. Calculated electric field in an asymmetric dualfrequency $27+2 \mathrm{MHz}$ reactor. The operating conditions are the same as in Fig. 4 applied for the symmetric reactor, i.e., applied HF and LF voltages of 200 and $400 \mathrm{~V}$, respectively, and a pressure of 30 mTorr. In the spherical model of the asymmetric reactor the PE is positioned at $5 \mathrm{~cm}$, the GE at $7 \mathrm{~cm}$, and the electrode ratio is 1.96 .

cycles. Other ions can be lost in electron detachment collisions [see Fig. 5(b)]. The time corresponding to $1 \mathrm{LF}$ ( $2 \mathrm{MHz}$ ) cycle is $5 \times 10^{-7} \mathrm{~s}$; hence the lifetime of $\mathrm{F}^{-}$in these cases is in the order of $5 \times 10^{-6} \mathrm{~s}$. In the dual-frequency reactor electron detachment can take place both in the bulk plasma and in the sheaths because the $\mathrm{F}^{-}$ions have high enough energy to participate in a reactive collision in the entire discharge. The calculated average energy is above $15 \mathrm{eV}$ in the bulk plasma and above $100 \mathrm{eV}$ in the sheaths. In this case the main loss mechanisms are the absorption at the electrodes and electron detachment collisions (see below). The $\mathrm{F}^{-}$density distribution is considerably modulated in the LF cycle [2] and negative ions are present in the sheaths not only because they are created in electron attachment collisions but also because they respond to the oscillating electric field.

The much heavier $\mathrm{CF}_{3}^{-}$ions, however, are still confined in the plasma and recombination is their major loss mechanism. The tracing of randomly sampled $\mathrm{CF}_{3}^{-}$ions is presented in Fig. 6. The ions are not yet lost after more than $200 \mathrm{LF}$ cycles; hence their lifetime is longer than $10^{-4} \mathrm{~s}$. The energy and $z$ velocity component are shown of the ion denoted (1). The energy of the other ion at the beginning rises up to $30 \mathrm{eV}$ but it is quickly dissipated in collisions with neutrals and then it has values close to the energy of the ion 1 . The $\mathrm{CF}_{3}^{-}$averaged energy in the bulk plasma is above $0.2 \mathrm{eV}$, i.e., higher than the calculated value of $0.04 \mathrm{eV}$ in the singlefrequency regime, due to the stronger electric field in the bulk plasma in the dual-frequency regime (see above).

As was found in Ref. [9] the sheath width decreases, and consequently the bulk plasma increases with the HF so that most $\mathrm{F}^{-}$ions gather less energy and cannot traverse the wide bulk plasma. In addition, a transition to electropositive behavior was observed, which means that the electric field in the bulk plasma is close to 0. Consequently, few ions can reach the electrodes when the applied HF increases from 27 to $100 \mathrm{MHz}$. Figure 7 presents the percentage of the $\mathrm{F}^{-}$ions lost at the electrodes, or by electron detachment or recombi- 

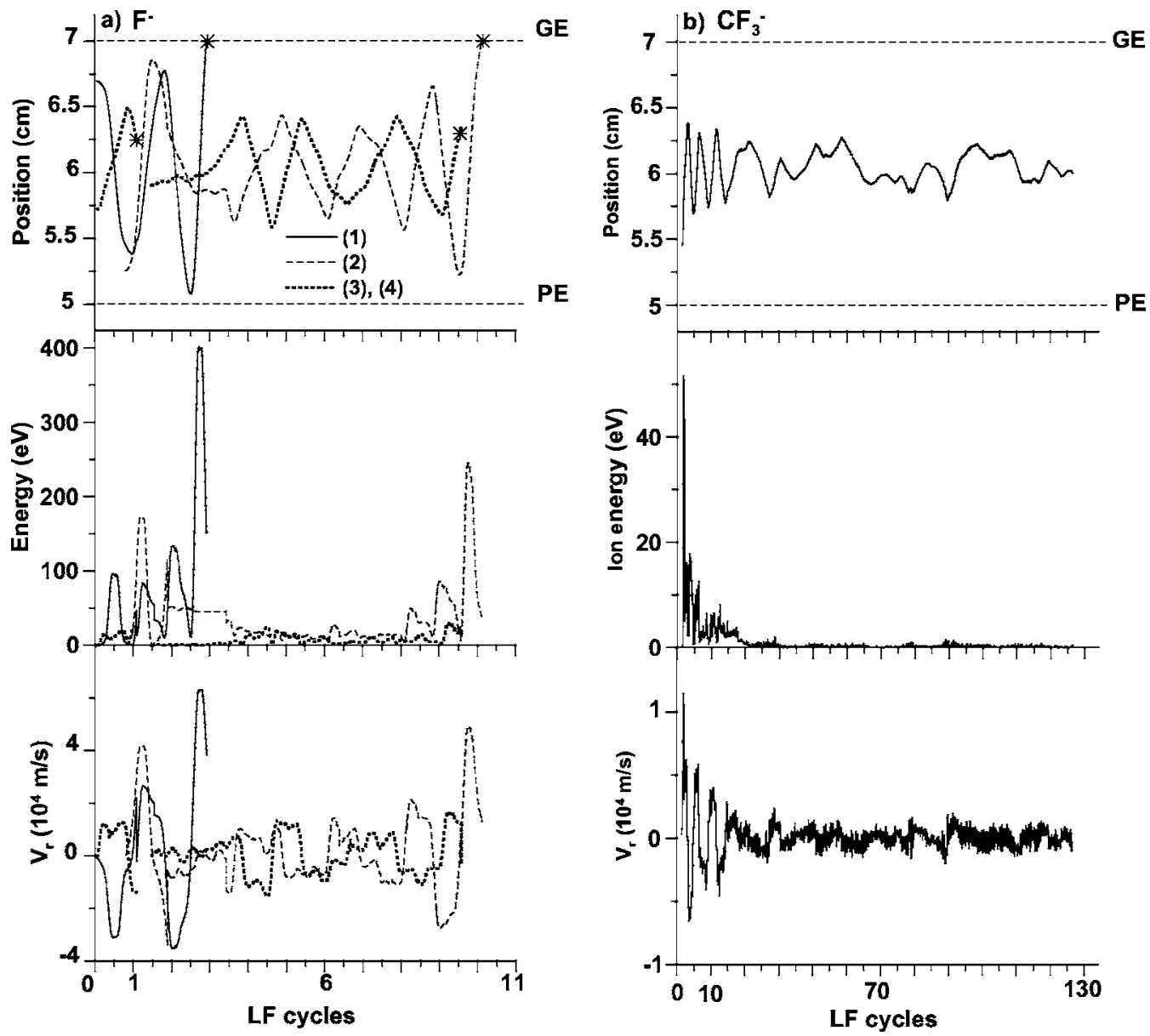

FIG. 11. Calculated position, energy, and $z$ velocity of randomly sampled $\mathrm{F}^{-}$ions (a) and $\mathrm{CF}_{3}^{-}$ion (b), created in electron attachment processes, as a function of time represented by LF cycles $\left(\tau_{L F}=5 \times 10^{-7} \mathrm{~s}\right)$. The $\mathrm{F}^{-}$ions 1 and 2 are lost at the electrodes, and ions 3 and 4 are lost in electron detachment collisions. The $\mathrm{CF}_{3}^{-}$ion has not been lost during the time of observation. The operating conditions are the same as in Fig. 10.

nation in the $27+2,40+2,60+2$, and $100+2 \mathrm{MHz}$ regimes, at applied $\mathrm{HF}$ and LF voltage amplitudes of 200 and $400 \mathrm{~V}$, respectively. The absorption at the electrodes and detachment collisions are the main loss mechanisms for the $27+2$ and $40+2 \mathrm{MHz}$ regimes. With increase of the $\mathrm{HF}$, recombination becomes the main loss mechanism. Hence, the $\mathrm{F}^{-}$ion flux at the electrodes is strongest for the operating conditions that give rise to a strongly electronegative discharge behavior and when the sheaths are comparatively wide.

The presented results explain the differences in the negative ion density distributions and calculated energies observed in single- and dual-frequency reactors $[2,6]$. In the single-frequency reactor $\mathrm{F}^{-}$and $\mathrm{CF}_{3}^{-}$ions are confined in the bulk plasma and $\mathrm{F}^{-}$ions represent the major negative charge because of the larger electron attachment cross section in comparison with the corresponding cross section for $\mathrm{CF}_{3}^{-}$ $[2,6]$. Their density distributions are constant throughout the rf cycle. Figure 8 shows their density profiles in the singlefrequency reactor for the same conditions, which are considered in Sec. III A. In the dual-frequency reactor under certain operating conditions (see above), the $\mathrm{F}^{-}$density distribution depends on the time in the LF cycle. Figure 9 presents the $\mathrm{F}^{-}$ density distribution as a function of time in 2 LF cycles (a) and averaged over $2 \mathrm{LF}$ cycles (b, dashed line), the $\mathrm{CF}_{3}^{-}$ density distribution ( $b$, solid line), the profile of which is constant in time, and the energy distribution of the $\mathrm{F}^{-}$ions lost at the electrodes (c). The presented characteristics are calculated for the $\mathrm{Ar} / \mathrm{CF}_{4} / \mathrm{N}_{2}$ discharge sustained in the $27+2 \mathrm{MHz}$ reactor, at $\mathrm{HF}$ and LF voltage amplitudes of 200 and $400 \mathrm{~V}$, respectively. The $\mathrm{F}^{-}$ions are lost mainly by fast electron detachment or by absorption at the electrodes, i.e., compare the lifetime in the order of $5 \times 10^{-6} \mathrm{~s}$ in these cases to the lifetime above $7 \times 10^{-5} \mathrm{~s}$ in the case of recombination. Hence, the $\mathrm{F}^{-}$density in the bulk plasma decreases to $5 \times 10^{13} \mathrm{~m}^{-3}$ for the considered operating conditions [Fig. 9(a)]. The $\mathrm{CF}_{3}^{-}$ions are still confined in the bulk plasma, their density distribution is constant throughout the LF cycle, and they represent the main negative charge with a density of $6 \times 10^{15} \mathrm{~m}^{-3}$ in the center [Fig. 9(b)]. The energy of the $\mathrm{F}^{-}$ ions absorbed at the electrodes can reach values of $200 \mathrm{eV}$ [Fig. 9(c)].

Finally, an asymmetric $\mathrm{Ar} / \mathrm{CF}_{4} / \mathrm{N}_{2}$ discharge, sustained in a $27+2 \mathrm{MHz}$ reactor, is considered. The applied $\mathrm{HF}$ and $\mathrm{LF}$ voltage amplitudes are 200 and $400 \mathrm{~V}$, respectively. A onedimensional spherical model is used to simulate the asymmetric discharge $[13,14]$. The inner $\mathrm{PE}$ is positioned at 
$r=5 \mathrm{~cm}$, and the GE at $r=7 \mathrm{~cm}$, so that the electrode area ratio is 1.96, and the electrode distance is $2 \mathrm{~cm}$ [14].

As was found in Refs. [14] and [16], the sheath voltage ratio is almost inversely proportional to the electrode area ratio. In the asymmetric discharge the dc bias voltage increases the sheath voltage in front of the smaller (powered) electrode; consequently the electric field is there much stronger than that in front of the GE [14]. Figure 10 presents the calculated electric field distribution in the asymmetric reactor as a function of time in two LF cycles. It can be seen that the maximum of the electric field at the PE is almost twice as high as the maximum at the GE, unlike in the symmetric reactor where the calculated electric field, presented in Fig. 4(a), reaches equal maximum values at the PE and GE. The electric field in the bulk plasma in both reactor types is comparatively strong and has maximum values of $10^{4} \mathrm{~V} / \mathrm{m}$. In addition the sheath in front of the PE is broader than the sheath in front of the GE [14]. Therefore, the $\mathrm{F}^{-}$ions reach mainly the GE because their energy is not high enough to reach the PE when they move from the bulk plasma to the $\mathrm{PE}$ and are retarded by the electric field.

The calculated position, energy, and $r$ velocity component of randomly sampled $\mathrm{F}^{-}$and $\mathrm{CF}_{3}^{-}$ions, as a function of time, represented by LF cycles, are shown in Figs. 11(a) and 11(b), respectively. All the ions are created in electron attachment collisions. The $\mathrm{F}^{-}$ions 1 and 2 are lost at the GE; ions 3 and 4 are lost in the electron detachment processes [Fig. 11(a)]. The $\mathrm{CF}_{3}^{-}$ions behave in the same way as in the symmetric discharge sustained in a dual-frequency reactor, i.e., they are confined in the plasma [Fig. 11(b)].

\section{SUMMARY}

Randomly sampled $\mathrm{F}^{-}$and $\mathrm{CF}_{3}^{-}$ions have been traced in single- and dual-frequency reactors, at different operating conditions, in both symmetric and asymmetric discharges.

It is shown that in symmetric or asymmetric singlefrequency reactors both types of negative ions are confined in the plasma and the loss mechanisms are recombination processes in the plasma and electron detachment in the sheaths. Comparatively high energies are calculated in the sheaths because the negative ions created by electron attachment collisions in the sheaths gain energy from the electric field on their way towards the bulk plasma.

In the dual-frequency reactor, however, in the presence of a LF, the light $\mathrm{F}^{-}$ions respond more or less to the applied electric field and can reach the electrodes. The $\mathrm{F}^{-}$energy is sufficiently high in the sheaths and plasma; hence electron detachment of $\mathrm{F}^{-}$can take place in the entire discharge. The absorption at the electrodes and electron detachment collisions are the main loss mechanisms. The negative $\mathrm{F}^{-}$ion flux at the electrodes is strongest when the operating conditions give rise to a strongly electronegative discharge behavior, and the sheaths are comparatively wide while the bulk plasma is narrow. The $\mathrm{F}^{-}$flux decreases when the applied HF increases because the sheath width decreases and the discharge exhibits more electropositive features, i.e., the electric field in the plasma is close to 0 . The much heavier $\mathrm{CF}_{3}^{-}$ions are still confined in the bulk but their average energy is calculated to be higher than in the single-frequency reactor.

In the asymmetric discharge, sustained in a dualfrequency reactor the $\mathrm{F}^{-}$ions reach mainly the GE because the electric field to the smaller electrode, i.e., PE, is much stronger than the electric field to the GE.

\section{ACKNOWLEDGMENTS}

The authors would like to thank R. Gijbels for helpful discussions. This research is supported by the Federal Services for Scientific, Cultural and Technical Affairs of the Prime Minister's Office through IUAP-V.
[1] M. A. Lieberman and A. J. Lichtenberg, Principles of Plasma Discharges and Materials Processing (John Wiley \& Sons, New York, 1994).

[2] V. Georgieva, A. Bogaerts, and R. Gijbels, J. Appl. Phys. 94, 3748 (2003).

[3] E. Gogolides, M. Stathakopoulos, and A. Boudouvis, J. Phys. D 27, 1878 (1994).

[4] N. V. Mantzaris, A. Boudouvis, and E. Gogolides, J. Appl. Phys. 77, 6169 (1995).

[5] K. Denpoh and K. Nanbu, J. Vac. Sci. Technol. A 16, 1201 (1998); , Jpn. J. Appl. Phys., Part 1 39, 2804 (2000).

[6] V. Georgieva, A. Bogaerts, and R. Gijbels, J. Appl. Phys. 93, 2369 (2003).

[7] K. Maeshige, G. Washio, T. Yagisawa, and T. Makabe, J. Appl. Phys. 91, 9494 (2002).
[8] T. Ohmori, T. K. Goto, T. Kitajima, and T. Makabe, Appl. Phys. Lett. 83, 4637 (2003).

[9] V. Georgieva and A. Bogaerts, J. Appl. Phys. 98, 023308 (2005).

[10] C. K. Birdsall and A. B. Langdon, Plasma Physics via Computer Simulation (McGraw-Hill, New York, 1985).

[11] C. K. Birdsall, IEEE Trans. Plasma Sci. 19, 65 (1991).

[12] V. Georgieva, A. Bogaerts, and R. Gijbels, Phys. Rev. E 69, 026406 (2004).

[13] M. A. Lieberman, J. Appl. Phys. 65, 4186 (1989).

[14] V. Georgieva and A. Bogaerts (unpublished).

[15] E. Kawamura, C. K. Birdsall, and V. Vahedi, Plasma Sources Sci. Technol. 9, 413 (2000).

[16] Y. Raizer, M. Shneider, and N. Yatsenko, Radio-Frequency Capacitive Discharges (CRC Press, Boca Raton, FL, 1995). 